\title{
Time and State Dependence in an Ss Decision Experiment
}

\author{
Jacopo Magnani, Aspen Gorry and Ryan Oprea
}

\section{ONLINE APPENDIX}

\section{A Theoretical Details}

\section{A.1 State Dependent Model and Solution Derivation}

Consider a firm that maximizes its flow profits at each moment by minimizing the expected discounted value of a quadratic instantaneous loss function:

$$
B\left(p-p^{*}\right)^{2}
$$

where $B$ is an exogenous constant parameter, $p$ is the current $(\log )$ price and $p^{*}$ is the target price, i.e. the price that maximizes instantaneous profits.

The target price follows a Brownian motion:

$$
d p^{*}(t)=\mu d t+\sigma d w(t)
$$

with drift $\mu$, volatility $\sigma$, where $w(t)$ is a standard Wiener process. The problem can be described entirely in terms of a single state variable: the price gap, $z=p-p^{*}$. The state variable follows a Brownian motion:

$$
d z(t)=-\mu d t+\sigma d w(t)
$$

For simplicity and to match the setting of our experiment, we restrict attention the case of $\mu=0$.

The firm's task is to decide whether to exert control over the state, $z$, by discontinuously adjusting it to the optimal level $z^{*}$ at each moment in time. In our simplified environment it is assumed that the optimal price $z^{*}$ is known (in the experiment $z^{*}$ is set equal to the optimal return point). The firm faces a fixed cost of $m$ each time it makes an adjustment and thus faces a sequence of optimal stopping problems: to choose the optimal times, $\left\{T_{i}\right\}$, to adjust the state to the optimal level, $z^{*}$, to optimize its value:

$$
v\left(z_{0}\right)=\min _{\left\{T_{i}\right\}} E_{0}\left\{\int_{0}^{T_{1}} e^{-r t} B z(t)^{2} d t+\sum_{i}\left[-e^{-r t} m+\int_{T_{i}}^{T_{i+1}} e^{-r t} B z(t)^{2} d t\right]\right\}
$$


The optimal solution to this problem is to establish an "inactivity band" for $z(t)$, $(-\bar{z}, \bar{z})$ : whenever $z(t)$ lies within the inactivity band the firm makes no adjustment and whenever $z(t)$ reaches or crosses these bounds, the firm pays $m$ to adjust $z(t)$ to $z^{*}$.

Inside the inaction region the value function can be characterized in the following way. Over a small time interval $\Delta t$, the following approximation holds:

$$
\begin{aligned}
v(z(t)) & =B z(t)^{2} \Delta t+\frac{1}{1+r \Delta t} E_{t} v(z(t+\Delta t)) ; \\
r \Delta t v(z(t)) & =B z(t)^{2}(1+r \Delta t) \Delta t+E_{t} v(z(t+\Delta t))-v(z(t)) ; \\
r v(z(t)) & =B z(t)^{2}(1+r \Delta t)+E_{t} \frac{v(z(t+\Delta t))-v(z(t))}{\Delta t}
\end{aligned}
$$

Taking the limit $\Delta t \rightarrow 0$ gives:

$$
r v\left(z(t)=B z(t)^{2}+\frac{1}{d t} E_{t} d v(z(t))\right.
$$

Using Ito's lemma it is possible to evaluate the expectation term:

$$
E_{t} d v(z(t))=\frac{1}{2} v^{\prime \prime}(z(t)) \sigma^{2} z^{2}(t) d t
$$

This leads to the Hamilton-Jacobi-Bellman equation:

$$
r v(z(t))=B z(t)^{2}+\frac{1}{2} \sigma^{2} v^{\prime \prime}(z(t))
$$

Requiring the value function to be symmetric around 0 and using standard techniques, it can be shown that the solution of this differential equation is:

$$
v(z(t))=\frac{B}{r} z(t)^{2}+B \frac{\sigma^{2}}{r^{2}}+C\left(e^{\frac{\sqrt{2 r}}{\sigma} z(t)}+e^{-\frac{\sqrt{2 r}}{\sigma} z(t)}\right)
$$

where $C$ is a constanst to be determined.

Outside the inaction region the value function is equal to the value at the optimal return point net of the menu cost. Thus the value of the firm over the entire state space is defined by:

$$
\begin{array}{ll}
v(z)=\frac{B}{r} z^{2}+B \frac{\sigma^{2}}{r^{2}}+C\left(e^{\frac{\sqrt{2 r}}{\sigma} z}+e^{-\frac{\sqrt{2 r}}{\sigma} z}\right) & z \in(-\bar{z}, \bar{z}) \\
v(z)=v\left(z^{*}\right)+m, & z \notin(-\bar{z}, \bar{z})
\end{array}
$$

The solution for the value function is obtained requiring that $v(z)$ and $v^{\prime}(z)$ are continuous at $\bar{z}$. These conditions are the following:

1. Value matching:

$$
v(\bar{z})=v\left(z^{*}\right)+m
$$


2. Smooth pasting:

$$
v^{\prime}(\bar{z})=0
$$

This system of two equations can be solved for $C$ and $\bar{z}$.

\section{A.2 Payoff Hills}

Here we plot the payoff hill for each treatment parametrization. The payoff hill plots the value $-v_{\bar{z}}\left(z^{*}\right)$ of the policy that uses a threshold $\bar{z}$ (not necessarily the optimal one) for adjusting, evaluated at the starting point $z^{*}$. This value function is computed using the value matching conditions.
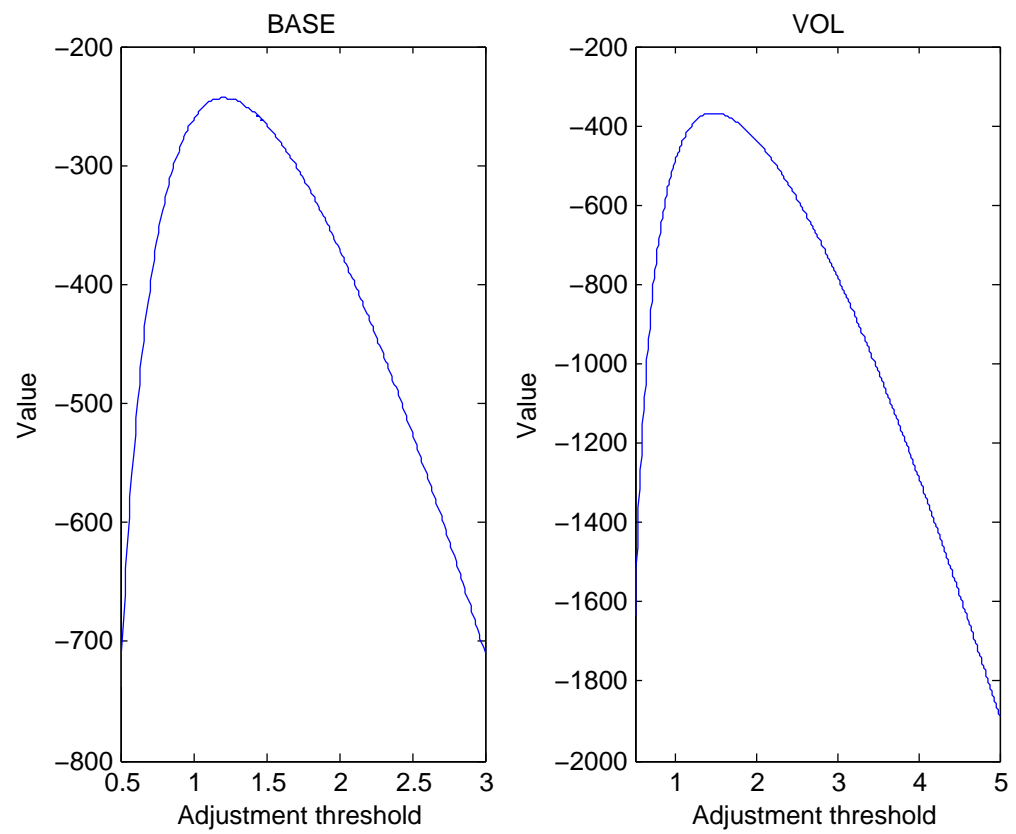

Figure 1: Payoff hills 


\section{B Instructions to Subjects}

\section{B.1 Instructions}

You are about to participate in an experiment in the economics of decision-making. The National Science Foundation and other agencies have provided the funding for this project. If you follow these instructions carefully and make good decisions, you can earn a CONSIDERABLE AMOUNT OF MONEY, which will be PAID TO YOU IN CASH at the end of the experiment.

Your computer screen will display useful information. Remember that the information on your computer screen is PRIVATE. To insure best results for yourself and accurate data for the experimenters, please DO NOT COMMUNICATE with the other participants at any point during the experiment. If you have any questions, or need assistance of any kind, raise your hand and one of the experimenters will come.

In the experiment you will make decisions over several periods.

At the end of the last period, you will be paid $\$ 5.00$, plus earnings based on your accumulated profits.

\section{B.2 The Basic Idea.}

You are in control of a company that sells its product at a Current Price. Your job is to decide when and how often to adjust the Current Price. Market conditions are constantly changing and so the Ideal Price - the price at which your current flow of profits would be maximized - is constantly changing. Each period of the experiment is a series of many ticks ( 5 ticks elapse each second). The relationship between your Current Price and the Ideal Price determines how much you earn each tick.

The further your Current Price is away from the Ideal Price (either too high or too low), the less money you make during the tick. If your Current Price is especially far from the Ideal Price you may even lose earnings during the tick. At any moment (and as often as you like) you will be allowed to pay a fixed cost, $\mathbf{C}$ (shown on the right side of your screen), to move your Current Price to the Ideal Price. At that point the Ideal Price will continue to fluctuate just as before, possibly moving away from the Current Price again.

\section{B.3 Screen Information.}

The screen is divided into three panels as shown in Figure 2.

The Top Panel shows you the Current Price in red and the fluctuating Ideal Price in blue. The rightmost tip (the leading edge) of each shows what is happening right now.

The Middle Panel helps you understand the consequences of your Current Price. It shows your Flow Profit, the amount you are earning (or losing) during each tick. When this is positive (and shaded in green) you are earning money; when it is negative (and shaded in red) you are actually losing accumulated money. By comparing the height of 

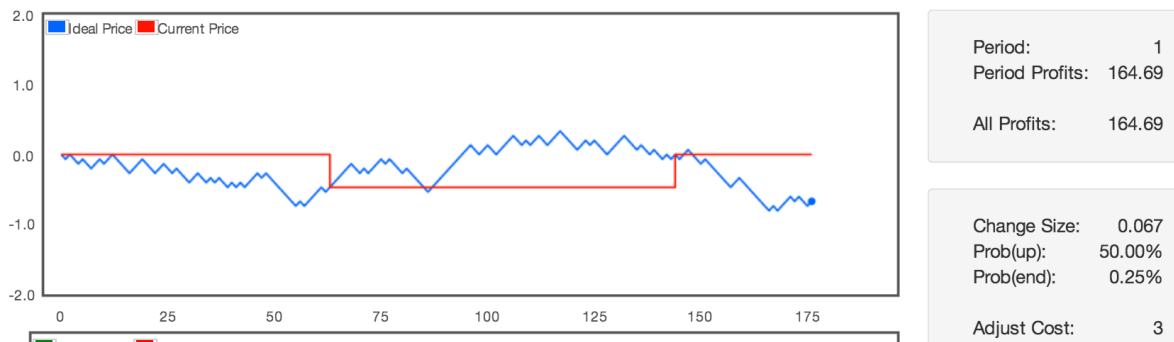

Adjust Cost: $\quad 3$
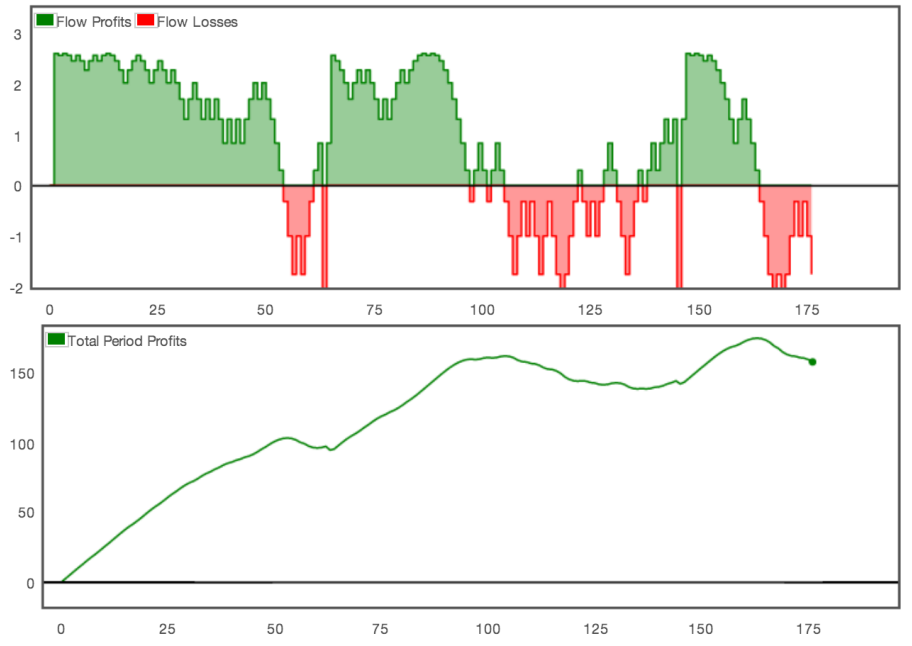

Figure 2: Screen example.

this line to the distance between Ideal Price and Current Price you can get a feeling for how differences between the two influence earnings.

The Bottom Panel shows you your Total Profits, the total amount of money you have accumulated so far during the period.

At any moment (and as often as you like) you can move your Current Price to the Ideal Price by pressing the spacebar. However remember that you will incur a significant cost each time you do this (that will show up in the Middle Panel and the Bottom Panel).

\section{B.4 Periods and Earnings.}

The experiment will be divided into a number of periods. The length of a period (in ticks) will be random - you will never know how long a period will last or when it is about to end (details below). At the beginning of a new period, the Current Price will automatically be realigned with the Ideal Price and your Period Profits will be reset to zero. (You can see your Period Profits on the right hand side of the screen.)

At the end of the experiment we will pay you for your total profits summed over all periods (shown as "All Profits" on the right side of the screen). This number will be 
converted to a payment amount according to the formula announced by the experimenter.

\section{B.5 Details.}

Here are a few more details on the experiment, in case you want to know:

Here are some details on how the Ideal Price unfolds:

- Each phase is a series of many "ticks" (5 ticks per second).

- Each tick the Ideal Price moves randomly up or down by a fixed amount, e.g., 0.75 point. The exact amount is shown on the right hand side of your screen as "Change Size."

- Upticks are as likely as downticks, e.g., each tick is up with probability $50 \%$ or down with probability 50\%. This is shown on the right side of your screen as "Prob(up)."

- The period ends with a small probability each tick, e.g., $\frac{1}{2}$ of $1 \%$. The exact amount is shown on the right hand side of your screen as "Prob(end)."

- The Current Price and the Ideal Price will always be identical at the beginning of each period.

Here are a few details on how the relationship between Current Price and the Ideal Price determines your earnings in each tick:

- Earnings are highest when your Current Price is equal to the Ideal Price and drop the further they are away from one another.

- Earnings are symmetric around the Ideal Price: you will earn just as much this tick if your Current Price is 1 point above the Ideal Price as if it is 1 point below the Ideal Price.

- The exact function that describes the relation between earnings in one tick, the Current Price and the Ideal Price is plotted in Figure ??. However you will get a good feel for this relationship from your screen over time. 


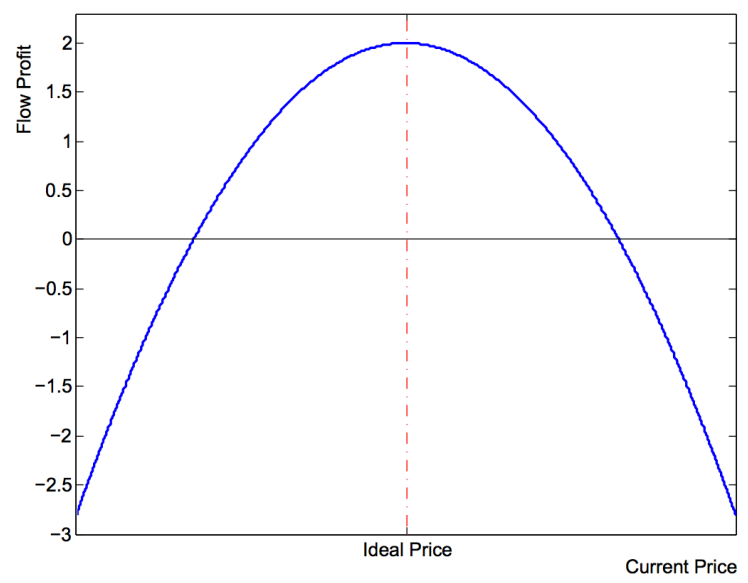

Figure 3: Earnings in a tick. Note: the red dashed line marks the Ideal Price. The blue line shows the level of your profits as the Current Price drops below or above this price.

\section{B.6 Frequently Asked Questions}

Q1. Is this some kind of psychology experiment with an agenda you haven't told us?

Answer: No. It is an economics experiment. If we do anything deceptive, or don't pay you cash as described, then you can complain to the campus Human Subjects Committee and we will be in serious trouble. These instructions are meant to clarify how you earn money, and our interest is in seeing how people make investment timing decisions.

Q2. How long does a phase last? Is there a minimum or maximum?

Answer: The length of time is random. In the example, the probability is 0.005 that any tick is the last, and there are 5 ticks per second. In this case, the average length of a round is 200 ticks or 40 seconds. Many rounds will last less than the average, and a few will last much longer. Rounds longer than 7 minutes are so unlikely that you probably will never see one. The minimum length is one tick, but it is unlikely you will ever see a round quite that short! 
Q3. How many rounds will there be?

Answer: Lots. We aren't supposed to say the exact number.

Q4. Are there patterns in upticks and downticks?

Answer: No. We've tried very hard to make it random. No matter what the recent history of upticks and downticks, the probability that the next tick is up is always the same (and is written on the board). 